Z. klin. Chem. u. klin. Biochem.

9. Jg., S. 39-42, Januar 1971

\title{
Elektrolytverschiebung und Serumenzyme Untersuchungen am Beispiel der experimentellen Tetrachlorkohlenstoffschädigung
}

\author{
Von H. KRöner, J. SefFen ${ }^{1}$ ) und W. Staib \\ Institut fïr Physiologisclie Chemie der Universität Diisseldorf
}

(Eingegangen am 5. Oktober 1970)

\begin{abstract}
Bei einer experimentellen Tetrachlorkohlenstoffvergiftung von Ratten wrurden Elektrolytbestimmungen in der Leber und Serumenzymbestimmungen durchgeführt. Die Natriumionenkonzentration in der Leber steigt kurzfristig an, etwas verzögert fällt die Kaliumionenkonzentration ab. Die Kinetik der Serumenzymaktivitäten ist für die untersuchten Enzyme unterschiedlich. Ein Vergleich mit der Kinetik der Elektrolytveränderungen in der Leber macht einen direkten Kausalzusammenhang zwischen diesen beiden Phänomenen der Tetrachlorkohlenstoffvergiftung unwahrscheinlich. Ergebnisse, die bei zusätzlicher Gabe von Cortisol erzielt werden, sprechen ebenfalls gegen einen Kausalzusammenhang zwischen Serumenzymveränderungen und Elektrolytverschiebungen in der Leber.
\end{abstract}

\section{Electrolyte sbift and serum enzymes in experimental carbon tetracbloride damage}

The liver electrolytes and serum enzymes of rats were determined during experimental carbon tetrachloride poisoning. The sodium ion concentration in the liver increased for a short period and the potassium ion concentration showed a somewhat delayed decrease. The kinetics of the serum enzyme activities varied depending on the enzymes studied. From a comparison of the kinetics of serum enzyme levels and of the variations of electrolytes in the liver, a direct causal relationship between these two phenomena seems improbable. Results obtained with the administration of cortisol also fail to show any indication of a causal relationship between serum enzyme changes and electrolyte shifts in the liver.

Die Folge einer Zellsschädigung ist in vielen Fällen ein Ausstrom von Enzymen aus der Zelle und damit ein Anstieg der Serumenzymaktivität. Die Schädigung kann dabei ganz unterschiedlicher Art sein, $z$. B. eine Ischämie beim Herzinfarkt, eine Infektion bei der Virushepatitis oder eine Intoxikation z. B. mit Tetrachlorkohlenstoff. Der Mechanismus des Enzymausstromes ist klar ersichtlich, wenn die Zellschädigung zu einer irreversiblen Zellzerstörung führt, z. B. zu einer Hämolyse der Exythrocyten. Für den Fall einer reversiblen Zellschädigung - und dazu zählen auch die Anfangsstadien einer später irreversiblen Schädigung - ist die Frage nach dem Mechanismus der Enzymausschüttung noch völlig offen. Zumindest konnte gezeigt werden $(1,2)$, daß nicht eine durch den gestörten Energiestoffwechsel gesteigerte Durchlässigkeit der Zellmembran für den Ausstrom verantwortlich ist.

Für den speziellen Fall der Leberzellschädigung durch Phalloidin konnten FrImMer und Mitarbeiter (3) einen Kaliumverlust nachweisen, der dem Ausstrom lysosomaler Enzyme aus der Leberzelle vorausgeht. Die Autoren halten einen kausalen Zusammenhang zwischen Ionenverminderungen und Enzymausstrom für wahrscheinlich. VertTy, Caper und Brown (4) haben eine Abhängigkeit der Bindung lysosomaler Enzyme von der Konzentration verschiedener Ionen nachgewiesen, und schließlich haben ARNold und PetTe (5) die ionenabhängige Bindung von Enzymen an Strukturproteine des Muskels beschrieben.

Es erscheint danach möglich, daß das Ionenmilieu beim Mechanismus der Enzymausschüttung generell eine
Rolle spielt. Zumal Ionenveränderungen im Zusammenhang mit der $\mathrm{CCl}_{4}$-Vergiftung beschrieben worden sind $(6,7)$, schien es uns interessant, diese Ionenveränderung und gleichzeitig die Serumenzymaktivitäten kinetisch zu verfolgen. Dabei haben wir uns nicht auf lysosomale Enzyme beschränkt.

In einer zweiten Versuchsserie haben wir den Einfluß von Cortisol auf die durch $\mathrm{CCl}_{4}$ verursachten Veränderungen untersucht. Entsprechend der klinischen Erfahrung, daß Cortisol die Serumenzymspiegel z. B. bei Hepatitis senkt, schien uns dieser Teil der Untersuchung sowohl im Hinblick auf den TetrachlorkohlenstoffWirkungsmechanismus als auch im Hinblick auf den Cortisolwirkungsmechanismus interessant.

\section{Methodik}

Versucbstiere waren männliche Wistarratten, 180 bis $220 \mathrm{~g}$ schwer, Firma Brünger, Bokel. Sie erhielten bis zu Versuchsbeginn Wasser und Standardfutter, Firma Höveler, Langenfeld-Immigrath.

Tetrachlorkohlenstoff p. a. Merck injizierten wir $1 \mathrm{ml}$ pro $\mathrm{kg}$ Körpergewicht in der vierfachen Menge Olivenöl gelöst intraperitoneal. $\mathrm{Zu}$ verschiedenen Zeiten nach der Tetrachlorkohlenstoff:njektion wurde ein Teil der Tiere in Âthernarkose entblutct und ein Stück Leber zur Untersuchung auf Natrium- und Kaliumionen entnommen. Dem anderen Teil der Tiere wurde in leichter Åthernarkose aus der Bauchaorta Blut entnommen, dessen Serum auf Lactatdehydrogenase (EC 1.1.1.27), saure Phosphatase (EC 3.1.3.2), $\beta$-Glucuronidase (EC 3.2.1.31) und Kathepsin untersucht wurde.

In einer weiteren Versuchsreihe wurden männliche Tiere sowohl mit Tetrachlorkohlenstof, $1 \mathrm{ml}$ pro $\mathrm{kg}$ Körpergewicht, als auch mit Hydrocortisonhemisuccinat, $50 \mathrm{mg}$ pro $\mathrm{kg}$ Körpergewicht,

1) Dissertation, Medizinische Fakultät der Universität Düsseldorf. 
behandelt. Die Injektion erfolgte wiederum intraperitoneal als 0,5 proz. Lösung in physiol. Natriumchloridlösung.

Die Bestimmung des Natrium- und Kaliumgebaltes der Leber erfolgte mit dem Flammenphotometer der Firma Eppendorf. Es wurden dazu 200 bis $300 \mathrm{mg}$ Gewebe nach der von Burck (8) beschriebenen Methode mit Schwefelsäure und Perhydrol verascht, anschließend mit Wasser verdünnt. Die Eichlösungen wurden aus einer Stammlösung hergestellt, die in einem Liter $1,5 \mathrm{mVal}$ $\mathrm{CaCO}_{3}, 12,5 \mathrm{mVal} \mathrm{NaCl}$ und $37,5 \mathrm{mVal} \mathrm{KCl}$ enthielt. Das Verhältnis der Kationenkonzentrationen in dieser Stammlösung und damit auch in den Eichlösungen entspricht etwa dem in der normalen Leber. Das Calciumcarbonat wurde zunächst unter Zusatz von etwas $1 \mathrm{~N} \mathrm{HCl}$ gelöst. Den Eichlösungen wurde konzentrierte Schwefelsäure in einer den veraschten Proben entsprechenden Menge zugesetzt.

Lactatdebydrogenase bestimmten wir nach Vorschrift der Firma Boehringer. Die Bestimmung der $\beta$-Glucuronidase erfolgte nach Frshmas (9). Als Substrat diente Phenolphthaleinglucuronid p. a., als Eichsubstanz Phenolphthalein der Firma Merck. Kathepsin bestimmten wir mit der von GIANETto und DeDuve (10) angegebenen Methode, bei einem $\mathrm{pH}$ von 3,6. Als Substrat diente Hämoglobin vom Rind nach Anson der Firma Serva, Heidelberg. Die säurelöslichen Tyrosin- und Tryptophanreste wurden nach Anson (11) mit dem Phenolreagenz von Folin-Ciocalteu bestimmt. Eichsubstanz war Tyrosin der Firma Schuchardt.

Die statistische A'uswertung erfolgte nach dem t-Test.

\section{Ergebnisse}

Innerhalb von 12 Stunden nach der Tetrachlorkohlenstoffinjektion kommt es zu einem kontinuierlichen Anstieg des Natriumgehaltes von $25 \mathrm{mVal}$ auf insgesamt $55 \mathrm{mVal}$ pro $\mathrm{kg}$ Lebergewebe (Abb. 1). Bereits zwei



Abb. 1

$\mathrm{Na}^{+-}$und $\mathrm{K}^{+-}$Gehalt der Leber in $\mathrm{mVal} / \mathrm{kg}$ Leberfrischgewicht. Zur Zeit 0 wurde $\mathrm{CCl}_{4}$, $1 \mathrm{ml} / \mathrm{kg}$ Körpergewicht in dem vierfachen Volumen mindestens 6 Tieren \pm Standardabweichung
Tind

Stunden nach der Injektion ist die Signifikanz des Natriumanstieges deutlich $(p<0,002)$, nach 12 Stunden ist $\mathrm{p}<0,001$.

In der gleichen Zeit sinkt der Kaliumgehalt des Lebergewebes. Nach sechs Stunden ist die Abnahme noch schwach $(\mathrm{p}<0,05)$, nach zwölf Stunden deutlich $(p<0,001)$ signifikant. Von der 4. Stunde nach Injektion fällt der Kaliumgehalt von $94 \mathrm{mVal}$ um 33\% auf $63 \mathrm{mVal}$ pro $\mathrm{kg}$ Lebergewebe nach 12 Stunden. Einem Kaliumverlust von $31 \mathrm{mVal}$ steht ein Natriumanstieg von $30 \mathrm{mVal}$ pro $\mathrm{kg}$ Lebergewebe 12 Stunden nach der Injektion gegenüber.

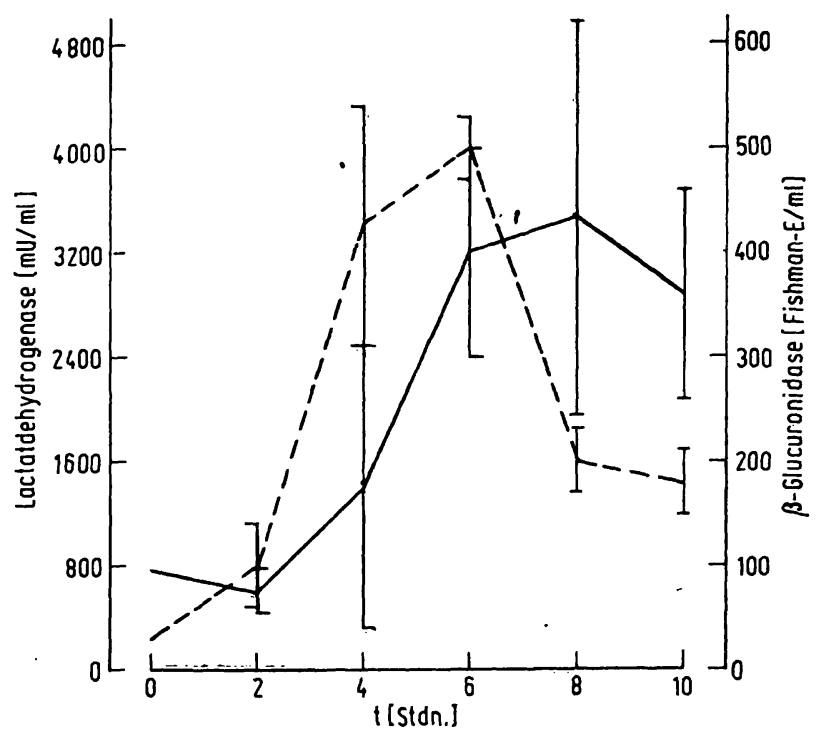

Abb. 2

Aktivität der Lactatdehydrogenase $(-)$ in $\mathrm{mU} / \mathrm{ml}$ und der $\beta$-Glucuronidase (- - ) in FISHMAN-Einheiten $/ \mathrm{ml}$ im Serum nach Gabe von $\mathrm{CCl}_{4}, 1 \mathrm{ml} / \mathrm{kg}$ Körpergewicht gelöst in dem vierfachen Volumen Olivenöl, i. p. injiziert. Eingezeichnet sind die Mittelwerte von mindestens 6 Tieren \pm Standardabweichung

Bereits 4 Stunden nach der Tetrachlorkohlenstoffvergiftung kommt es zu einer fast 100proz. Steigerung der Lactatdehydrogenase-Aktivität im Serum, und zwar von 753 auf $1428 \mathrm{mU} / \mathrm{ml}$ (Abb. 2). Nach weiteren 2 Stunden stellt sich ein Maximum ein, bei dem das Vierfache des Ausgangswertes erreicht ist $(p<0,001)$. Auf dieser Höhe hält sich die Enzymaktivität über längere Zeit. Auch 10 Stunden nach der Injektion beträgt sie noch $2900 \mathrm{mU} / \mathrm{ml}$.

Der Kontrollwert der $\beta$-Glucuronidase im Serum beträgt 36 Fishman-Einheiten $/ \mathrm{ml}$. 2 Stunden nach der Injektion kommt es zu einem signifikanten Aktivitätsanstieg $(p<0,01)$ auf fast 100 Einheiten $/ \mathrm{ml}$. 6 Stunden nach der Vergiftung zeigt sich ein Maximum der Aktivität, bei dem das Zwölffache des Ausgangswertes erreicht ist $(p<0,001)$. Innerhalb der nächsten 2 Stunden kommt es dann zu einem steilen Abfall der Aktivität bis auf das Fünffache des Kontrollwertes. Danach setzt sich der Aktivitätsverlust langsam um 20 Einheiten $/ \mathrm{m} l$ in 2 Stunden fort. Die Enzymaktivität ist aber auch nach 10 Stunden noch viermal so hoch wie der Ausgangswert.

Die saure Phosphatase im Serum unbehandelter Tiere beträgt $19 \mathrm{mU} / \mathrm{ml}$. 4 Stunden nach der Vergiftung kommt es zu einem leichten Anstieg der Aktivität ( $p<0,05$ ) (Abb. 3), nach 8 Stunden beträgt die Steigerung der Aktivität mehr als $130 \%$ ( $p<0,001)$. Danach kommt es bis zur 10. Stunde nach der Injektion zu einem leichten Aktivitätsrückgang bis auf $36 \mathrm{mU} / \mathrm{ml}$. Auch die Aktivität von Kathepsin im Serum steigt nur langsam an, und $z$ war von 5,1 auf $1.1,4 \mathrm{mU} / \mathrm{ml}$ nach 8 Stunden $(p<0,001)$. Bereits in den folgeriden 2 Stunden nimmt die Aktivität um 40\% ab.

In einer zweiten Versuchsreihe erhalten die Tiere gleichzeitig Tetrachlorkohlenstoff und Cortisol, die Kontrollen erhalten statt Cortisol physiol. NaCl-Lösung. Auch bei diesen Versuchen steigt der Natriumgehalt 


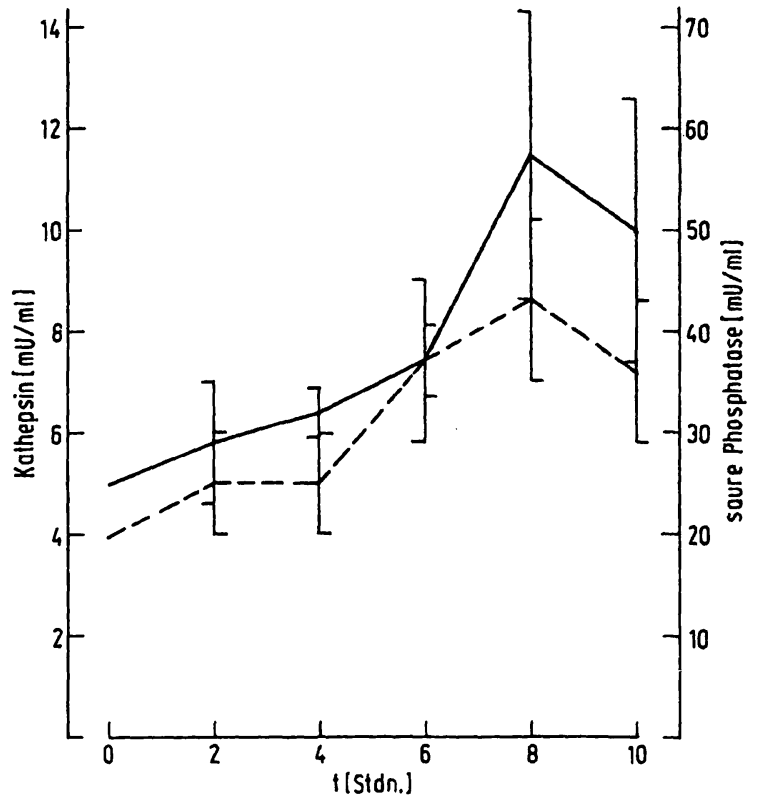

Abb. 3

Aktivität von Kathepsin $(-)$ und saurer Phosphatase $(--)$ in $\mathrm{mU} / \mathrm{ml}$ im Serum nach Gabe von CCla, $1 \mathrm{ml} / \mathrm{kg}$ Körpergewicht gelöst in dem vierfachen Volumen Olivenöl, $i$. p. injiziert. Eingezeichnet sind die
Mittelwerte von mindestens 6 Tieren \pm Standardabweichung

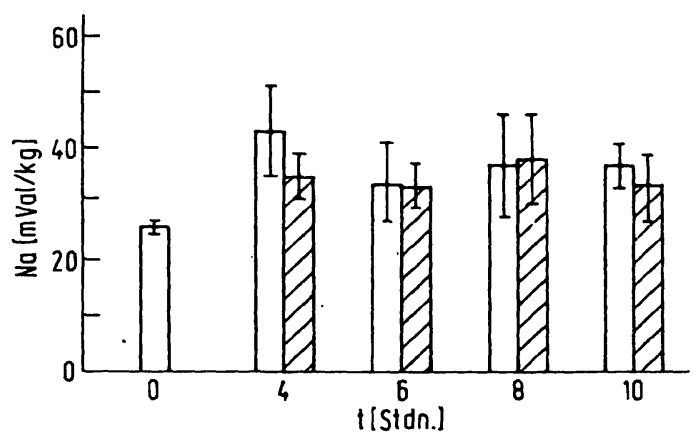

Abb. 4

$\mathrm{Na}+-$ Gehalt der Leber in $\mathrm{mVal} / \mathrm{kg}$ Frischgewicht. Zur Zeit 0 wurde $\mathrm{CCl}_{4}, 1 \mathrm{ml} / \mathrm{kg}$ Körpergewicht in dem vierfachen Volumen Olivenöl gelöst, i. p. injiziert. Schraffierte Säulen: Zusätzlich wurde gleichzeitig Cortisolhemisuccinat, $50 \mathrm{mg}$ Cortisol/kg Körpergewicht, i. p. succinat physiol. $\mathrm{NaCl}$-Lösung. Mittelwerte von je 6 Experimenten + Standardabweichung

der Leber signifikant bis zur 4. Stunde an (Abb. 4), bleibt dann aber etwa auf der gleichen Höhe. Gegenüber den mitgeführten Kontrollen ergeben sich kaum Differenzen. Der $\mathrm{K}^{+}$-Gehalt der Leber fällt bei gleichzeitiger Injektion von Cortisol und Tetrachlorkohlenstoff nur geringfügig ab (Abb. 5), der Wert 4 Stunden nach der Injektion unterscheidet sich signifikant von den unbehandelten Kontrollen $(p<0,05)$. Gegenüber Kontrollen, die neben Tetrachlorkohlenstoff nur $\mathrm{NaCl}$ erhielten, sind keine signifikanten Differenzen festzustellen.

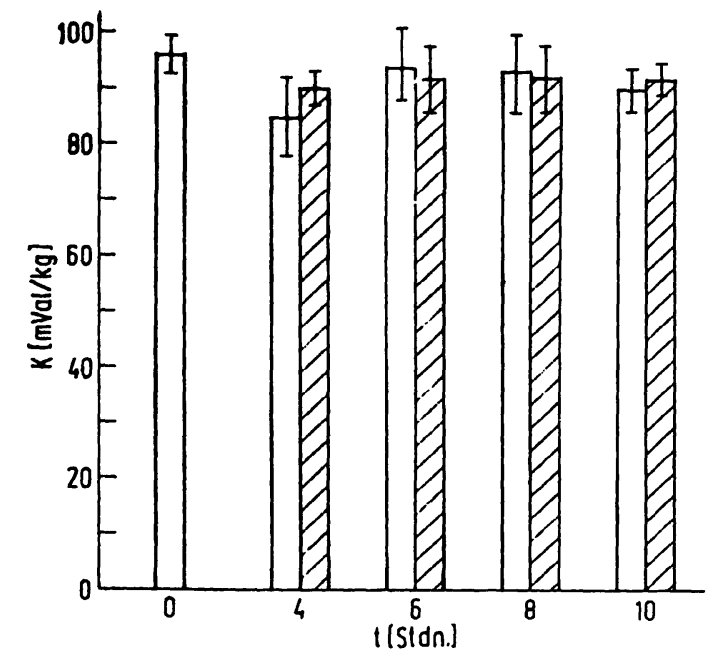

Abb. 5

$\mathrm{K}+-$ Gehalt der Leber in $\mathrm{mVal} / \mathrm{kg}$ Frischgewicht. Zur Zeit 0 wurde $\mathrm{CCl}_{1}, 1 \mathrm{ml} / \mathrm{kg}$ Körpergewicht in dem vierfachen Volumen Olivenöl gelöst, i. p. injiziert. Schraffierte Säulen: Zusätzlich wurde gleichzeitig Cortisolhemisuccinat, $50 \mathrm{mg}$ Cortisol/kg Körpergewicht, i. p. injiziert. Leere Säulen: Kontrollen, erhielten statt Cortisolhemisuccinat physiol. $\mathrm{NaCl}$-Lösung. Mittelwerte von je 6 Experimenten Standardabweichung

Die Enzymveränderungen sind bei gleichzeitiger Injektion von Tetrachlorkohlenstoff und Cortisolhemisuccinat nur gering (Tab. 1). So nimmt die Lactatdehydrogenase-Aktivität des Serums nur um etwa 25\% nach 6 Stunden zu, die Differenz ist nicht signifikant. Der Anstieg der $\beta$-Glucuronidaseaktivität ist dagegen deutlich und signifikant und von der gleichen Größenordnung wie bei Tieren, die nur Tetrachlorkohlenstoff erhalten haben. Die saure Phosphatase im Serum bleibt nach der Injektion von Tetrachlorkohlenstoff und Cortisol unverändert gegenüber unbehandelten Normaltieren. Die Kathepsinaktivität steigt etwas an, um 30\% nach 4 Stunden und um 50\% im Mittel nach 6 Stunden. Der letzte Wert ist auch signifikant $(p<0,001)$ gegenüber den unbehandelten Kontrollen, liegt aber niedriger als der entsprechende Wert in der ersten Versuchsserie ohne Cortisol.

\section{Diskussion}

Verglichen mit dem von FrIMMER und Mitarbeitern (3) beschriebenen Kaliumausstrom aus der mit Phalloidin vergifteten Rattenleber, ist der Kaliumverlust bei der Tetrachlorkohlenstoffvergiftung deutlich verzögert. Der Abfall der Kaliumkonzentration in der Leber beginnt erst 6 Stunden nach der $\mathrm{CCl}_{4}$-Applikation und stimmt damit zeitlich gut überein mit dem Abfall der energiereichen Phosphate, wie er früher beschrieben wurde (13). Es liegt nahe, hier auch einen kausalen $\mathrm{Zu}$ -

Tab. 1

Serumenzymaktivitäten im Serum nach Gabe von $\mathrm{CCl}_{4}, 1 \mathrm{ml} / \mathrm{kg}$ Körpergewicht gelöst in vierfachem Volumen Olivenöl. Ein Teil der Tiere erhielt zusätzlich Cortisolnten Tieren \pm Standardabweichung

\begin{tabular}{|c|c|c|c|c|c|}
\hline Vorbehandlung & $\begin{array}{l}\text { Zeit nach Applikation } \\
\text { [Stdn.] }\end{array}$ & $\begin{array}{l}\text { Kathepsin } \\
{[\mathrm{mU} / \mathrm{ml}]}\end{array}$ & $\begin{array}{l}\text { saure Pliosphatase } \\
{[\mathrm{mU} / \mathrm{ml}]}\end{array}$ & $\begin{array}{c}\beta \text {-Glucuronidase } \\
{[\text { FisHMAN-Einheiten/ml] }}\end{array}$ & $\begin{array}{l}\text { Lactatdehydrogenase } \\
{[\mathrm{mU} / \mathrm{ml}]}\end{array}$ \\
\hline $\begin{array}{l}\mathrm{CCl}+\mathrm{NaCl} \\
\mathrm{CCl}+\text { + Cortisol } \\
\mathrm{CCl}+\mathrm{NaCl} \\
\mathrm{CCl}_{4}+\text { + Cortisol }\end{array}$ & $\begin{array}{l}4 \\
4 \\
6 \\
6\end{array}$ & $\begin{array}{l}6,4 \pm 0,6 \\
6,0 \pm 0,5 \\
7,4 \pm 0,7 \\
6,8 \pm 1,1\end{array}$ & $\begin{array}{l}26,1 \pm 5,8 \\
20,8 \pm 2,4 \\
36,5 \pm 7,7 \\
18,9 \pm 3,6\end{array}$ & $\begin{array}{l}425 \pm 114 \\
279 \pm 51 \\
505 \pm 60 \\
297 \pm 149\end{array}$ & $\begin{array}{r}1428 \pm 1110 \\
757 \pm 267 \\
3191 \pm 733 \\
934 \pm 193\end{array}$ \\
\hline
\end{tabular}


sammenhang anzunehmen. Die in der Literatur bei der $\mathrm{CCl}_{4}$-Intoxikation beschriebenen Ionenveränderungen $(6,7)$ liegen zu einem Zeitpunkt, wo nach unseren Ergebnissen (13) ebenfalls der Energiestoffwechsel bereits gestört sein dürfte. Anders verhält sich die $\mathrm{Na}^{+}$ Konzentration in der Leber nach Gabe von Tetrachlorkohlenstoff. Der sofort einsetzende Anstieg könnte bedingt sein durch Veränderungen des extrazellulären Raumes, im Sinne eines entzündlichen Ödemes. Obwohl wir keine nennenswerten Unterschiede des Trockengewichtes gegenüber Kontrollen gefunden haben, sind ödematöse Veränderungen und erhöhter Blutgehalt nicht sicher auszuschließen.

Der Anstieg der untersuchten Serumenzyme ist insgesamt gesehen nicht so massiv, da für die Versuche männliche Tiere verwendet wurden (14). Wir versprachen uns davon den Vorteil, zeitliche Zusammenhänge bei einer mäßigen Schädigung besser erkennen $z u$ können als bei totaler Zerstörung der Leber. So bestehen allein $z$ wischen den Aktivitätszunahmen der untersuchten Serumenzyme deutliche zeitliche Differenzen. Der steile Anstieg der $\beta$-Glucuronidase erfolgt bis zur 4. Stunde nach $\mathrm{CCl}_{4}-\mathrm{Gabe}$ und liegt damit sicher vor dem Kaliumausstrom. Die hauptsächliche Zunahme der Serum-Lactatdehydrogenase liegt $z$ wischen 4 und 6 Stunden nach $\mathrm{CCl}_{4}$-Gabe, und damit unseres Erachtens auch noch zu früh, als daß sie Folge des Kaliumionenverlustes sein könnte. Das Maximum der Kathepsinund Phosphataseaktivität im Serum liegt dagegen erst nach 8 Stunden, der Anstieg ist relativ gering. Gegen einen kausalen Zusammenhang zwischen Kaliumverlust der Leber und Enzymaustritt, wie er von FrImMer und Mitarbeitern (3) für die Phalloidinvergiftung postuliert wird, spricht auch die Beobachţung, daß die Serumenzymaktivitäten nach $6 \mathrm{bzw} .8$ Stunden nicht weiter ansteigen, obwohl die Lebern weiterhin Kaliumionen verlieren. Die von uns beobachtete Kalium-NatriumVerschiebung in der Leber scheint lediglich eine Folge des gestörten aktiven Transportes infolge des gestörten Energiestoffwechsels zu sein. Wir konnten schon früher zeigen, daß eine Störung des Energiestoffwechsels nicht eine Enzymfreisetzung bedingt $(13,14)$.

Durch die gleichzeitige Gabe von Cortisolhemisuccinat werden die Ionenveränderungen der Leber durch Tetrachlorkohlenstoff weitgehend aufgehoben. $\mathrm{Da}$ aber auch die alleinige Verabfolgung von $\mathrm{NaCl}$ als Kontrolle den gleichen Effekt zeigt, könnte auch eine gestörte Resorption des $\mathrm{CCl}_{4}$ der Grund für die verhinderten Ionenveränderungen sein. Gegen diese Annahme spricht der Anstieg der $\beta$-Glucuronidase im Serum, der nach zusätzlicher Cortisolgabe etwa von der gleichen Größenordnung ist wie nach alleiniger $\mathrm{CCl}_{4}$-Verabfolgung. Das ist insofern interessant, als gerade die Lysosomen durch Cortisol stabilisiert werden sollen $(15,16)$. Der Anstieg der übrigen Serumenzymaktivitäten ist dagegen bei zusätzlicher Cortisolgabe vergleichsweise gering. $\mathrm{Ob}$ das ein spezifischer Effekt ist (17), müssen weitere Untersuchungen klären.

\section{Literatur}

1. KRÖNER, H. und W. StaIB, diese Z. 6, 318 (1968). - 2. KRÖNER, H. und W. StaIB, diese Z. 7, 175 (1969). - 3. Frimmer, M., J. Gries und D. Hegener, Naunyn-Schmiedebergs Arch. exp. Pathol. Pharmakol 258, 197 (1967). - 4. Verty, M. A., R. CAPER und W. J. Brown, Biochem. J. 109, 149 (1968). - 5. ARNoLd, H. und D. Pette, Europ. J. Biochem. 6, 163 (1968). - 9. Rees, K. R., in Enzymes and Drug Action in Ciba Found. Sympos. Edit. J. L. Mongar and A. V. S. de Reuck, Little, Brown \& Co. Boston (1962). - 7. Thiers, R. E., E. S. Reynolds und B. L. ValleE, J. biol. Chemistry 235, 2130 (1960). - 8. Burck, H. C., Klin. Wschr. 39, 751 (1961). - 9. Frshman, W. H., in Methoden der enzymatischen Analyse, Hrsg. H. U. Bergmeyer, Verlag Chemie GmbH, Weinheim/Bergstr. (1962). - 10. Gianetro, R. und C. DeDuve, Biochem. J. 59, 433 (1955). - 11. Asson, M. L., J. Gen. Physiol. 20, 565 (1937). - 12. Martin, J. B. und D. M. Doty, Zit. nach H. Weil-Malherbe, in Hoppe-Seyler-Thierfelder, Handbuch Physiol Pathol. Chem. Analyse III, Hrsg. K. Lang und E. Lehnartz, Springer-Verlag, Berlin, Göttingen, Heidelberg (1955). - 13. KRöNER, H. und W. STAIB, Hoppe Seyler's Z. physiol. Chem. 348, 575 (1967). - 14. KRöNER, H. und W: STAIB, diese Z. 5, 89 (1967). - 15. WeissmanN, G. und L. Thomas, J. Clin. Invest. 42, 661 (1963). - 16. WeIssmanN, G. und L. Thomas, Recent Progr. Hormone Res., N. Y. 20, 215 (1964). - 17. TAKEDA, Y., A. Achikara, H. Tanioka und H. Inone, J. biol. Chemistry 239,3590 (1964).

Prof. Dr. W. Staib Physiol.-chem. Inst. 4000 Düsseldorf 1 Witzelstr. 111 